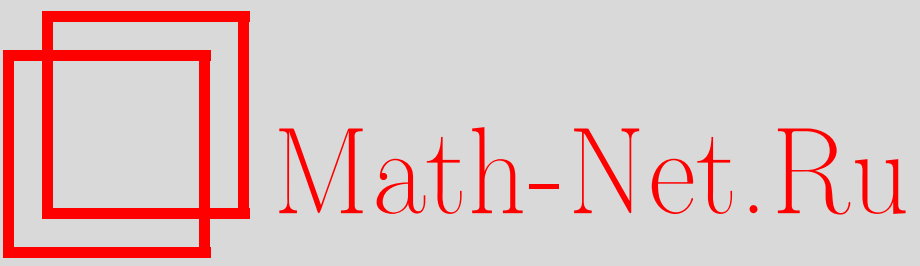

Д. В. Ширков, Аналитическая теория возмущений для наблюдаемых квантовой хромодинамики, ТМФ, 2001, том 127, номер 1, 3-20

DOI: https://doi.org/10.4213/tmf445

Использование Общероссийского математического портала Math-Net.Ru подразумевает, что вы прочитали и согласны с пользовательским соглашением

http://www.mathnet.ru/rus/agreement

Параметры загрузки:

IP: 3.80 .181 .102

26 апреля 2023 г., 13:42:04 
ТЕОРЕТИЧЕСКАЯ

И МАТЕМАТИЧЕСКАЯ

ФИЗИКА

Том 127, № 1

апрель, 2001

(C) 2001 г.

\section{АНАЛИТИЧЕСКАЯ ТЕОРИЯ ВОЗМУЩЕНИЙ ДЛЯ НАБЛЮДАЕМЫХ КВАНТОВОЙ ХРОМОДИНАМИКИ}

Исследуется связь между свободными от "призрачных" сингулярностей ренорминвариантными разложениями теории возмущений КХД во времени- и пространственноподобных областях. Основным средством является "двойное спектральное представление" (подобное представлению для функции Адлера), вытекающее из основных аксиом локальной КТП и связывающее между собой действительные функции в евклидовой и минковской (т.е. времениподобной) областях. На этой основе установлено простое соответствие между известным с начала 80 -х годов приемом суммирования $\pi^{2}$-членов в инвариантную функцию связи $\tilde{\alpha}(s)$ и наблюдаемые КХД во времениподобной области и развитым несколько лет назад инвариантным аналитическим подходом, приводящим к свободным от нефизических сингулярностей "аналитизированным" инвариантной функции связи $\alpha_{\mathrm{an}}\left(Q^{2}\right)$ и нестепенным разложениям для наблюдаемых в пространственноподобной области. Сформулированы самосогласованная схема, аналитическая теория возмущений (АТВ), связывающая между собой ренорминвариантные эффективные функции связи $\alpha_{\mathrm{an}}\left(Q^{2}\right)$ и $\tilde{\alpha}(s)$, а также получены нестепенные разложения теории возмущений для наблюдаемых в евклидовой и минковской областях, свободные от нефизических сингулярностей и отличающиеся улучшенной сходимостью в инфракрасной области. Проведено "глобальное" обобщение новой схемы АТВ на случай реальной КХД, включающий области с различным числом активных кварков. Предварительные оценки показывают, что вычисления в рамках глобальной АТВ могут приводить к результатам, заметно отличающимся от обычных расчетов для $\bar{\alpha}_{s}$ даже в пятикварковой области. Приведены численные примеры.

\section{СОДЕРЖАНИЕ}

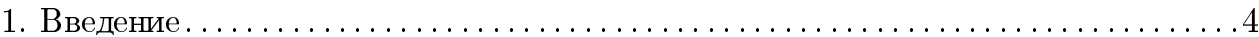

1.1. Преамбула: теория возмушений и параметр степенного разложения $\bar{\alpha}_{s} . .4$

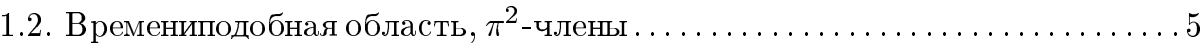

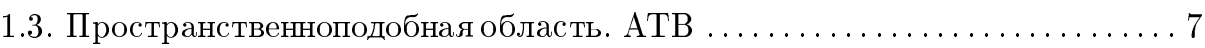

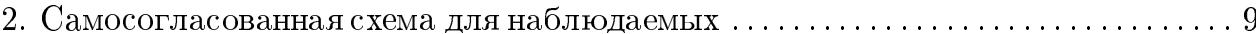

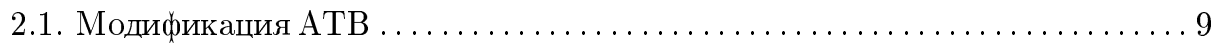

* Лаборатория теоретической физики им. Н.Н. Боголюбова, ОИЯИ, Дубна, Московская обл., Россия. E-mail: shirkovd@thsun1.jinr.ru 
2.2. Разложения по последовательностям $\{\mathcal{A}\}$ и $\{\mathfrak{A}\} \ldots \ldots \ldots \ldots \ldots \ldots \ldots$

3. Глобальная формулировка АТВ .............................. 12

3.1. Сопряжение на порогах . . . . . . . . . . . . . . . . . . . . 13

3.2. $s$-Канал. Константы сдвига.............................. 14

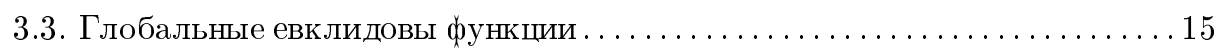

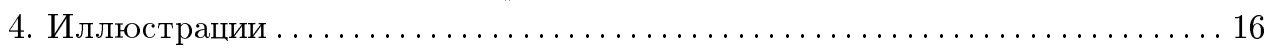

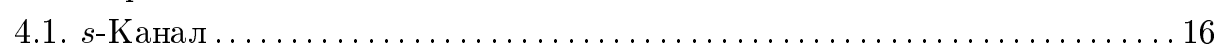

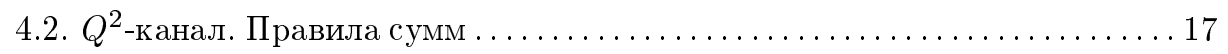

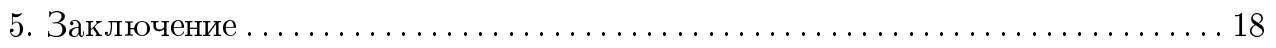

\section{1. ВВЕДЕНИЕ}

1.1. Преамбула: теория возмущений и параметр степенного разложения $\bar{\alpha}_{s}$. Вопросы поведения сильного взаимодействия в области средних и низких энергий $W=\sqrt{s}$ и передач импульса $Q=\sqrt{Q^{2}}$ благодаря быстрому накоплению экспериментальных данных постоянно привлекают повышенный интерес теоретиков. В качестве основного средства анализа здесь используется пертурбативная квантовая хромодинамика, несмотря на то что в указанной области параметр степенного разложения $\alpha_{s}$ перестает быть “достаточно малой” величиной. Физически эта область отвечает трем $(f=3)$ и четырем $(f=4)$ активным кваркам. Именно в трехкварковой области располагаются нефизические особенности основной теоретической величины - инвариантной функции связи $\bar{\alpha}_{s}$, характеризуемые так называемым параметром шкалы КХД $\Lambda_{f=3} \simeq 350$ МэВ. Эти особенности, наличие которых, кстати сказать, противоречит основным принципам локальной КТП, сушественно осложняют теоретическую интерпретацию данных в области низких энергий и малых передач импульса $\left(W, Q \lesssim 3 \Lambda_{3}\right)$.

Следует отметить, что в текушей литературе для эффективной функции связи КХД во времениподобной области $\alpha_{s}(s), s=W^{2}$, используют буквально то же самое выражение, что и в евклидовой области, тем самым перенося нефизические сингулярности в трехкварковую область низких энергий $W \simeq 350 \mathrm{MэВ.}$

Между тем понятие (квадрата) инвариантного заряда электрона $\bar{\alpha}\left(Q^{2}\right)=\bar{e}^{2}(Q)$ в КЭД изначально было определено в работах [1] по ренормгруппе (РГ) лишш в пространственноподобной области через произведение действительных констант $z_{i}$ конечных перенормировочных преобразований Дайсона. Именно евклидов заряд $\bar{e}(Q)$ связан преобразованием Фурье с пространственным распределением электрического заряда $\bar{e}(r)$ вокруг затравочного электрона, введенного еше Дираком [2]. Аналогичное обоснование в формализме РГ (детали см. в главе "Ренормализационная группа" книги [3]) имеет инвариантная функция связи $\bar{g}(Q)$, определенная лишь в евклидовой области. Никакого естественного простого определения для функции $\bar{g}$ в “минковской” (т.е. времениподобной) области из аппарата РГ не следует.

Тем не менее современная практика, ориентируюшаяся на "освяшенные авторитетом" высокие обзоры (см., например, [4], [5] и монографию [6]), использует одно и то же сингулярное выражение для эффективной функции связи КХД $\bar{\alpha}_{s}$ как в пространственно-, так и во времениподобной областях. 
Технически во времениподобной области такое "заимствование" евклидова функционального вида эффективной функшии связи сопровождается модификашией численных коэффишиентов пертурбативного разложения. К коэффишиенту, вычисляемому по соответствуюшим вкладам от диаграмм Фейнмана, добавляются специфические (пропорциональные числу $\pi^{2}$ и его степеням) вклады с коэффициентами от более низких членов разложения. Этими $\pi^{2}$-вкладами и исчерпьвается "расплата за переправу через Стикс" из царства Евклида в мир Минковского.

1.2. Времениподобная область, $\pi^{2}$-члены. Как нетрудно показать, подобная процедура основана на малости параметра $\pi^{2} / \ln ^{2}\left(s / \Lambda^{2}\right)$. Поэтому она оказывается оправданной лишш в области достаточно высоких энергий $W \gg \Lambda e^{\pi / 2} \simeq 3$ ГэВ. В этой связи полезно обратиться к идеям, развитым в начале 80-х годов Радюшкиным [7] и Красниковым и Пивоваровым [8] (процедура РКП). Для введения инвариантной функции $\bar{\alpha}_{s}$ во времениподобной области ими было использовано интегральное преобразование $\mathbf{R}$, обратное дипольному преобразованию $\mathbf{D}$ полного сечения в функцию Адлера. Последнее может быть рассмотрено как определение интегральной операции

$$
R(s) \rightarrow D(z)=Q^{2} \int_{0}^{\infty} \frac{d s}{(s+z)^{2}} R(s) \equiv \mathbf{D}\{R(s)\},
$$

преобразующей действительную функцию $R(s)$ положительного (времениподобного) аргумента в функцию $D(z)$, определенную в комплексной плоскости с разрезом, точнее $\mathrm{c}$ аналитическими свойствами, отвечающими интегральному представлению Челлена-Лемана. В частности, функция $D\left(Q^{2}\right)$ является действительной на действительной положительной полуоси $z=Q^{2}+i 0, Q^{2} \geqslant 0$.

Обратная операция $\mathbf{R}$ может быть представлена через контурный интеграл:

$$
R(s)=\frac{i}{2 \pi} \int_{s-i \varepsilon}^{s+i \varepsilon} \frac{d z}{z} D_{\mathrm{pt}}(-z) \equiv \mathbf{R}\left[D\left(Q^{2}\right)\right]
$$

$\mathrm{C}$ помошью операции $\mathbf{R}$ можно определить эффективную ренорминвариантную функцию связи $\tilde{\alpha}(s)=\mathbf{R}\left[\bar{\alpha}_{s}\left(Q^{2}\right)\right]$ во времениподобной области. Опуская технические подробности, приведем несколько простых результируюших выражений.

1. Для однопетлевой функции связи $\bar{\alpha}_{s}^{(1)}=\left[\beta_{0} \ln \left(Q^{2} / \Lambda^{2}\right)\right]^{-1}$ находим $[9],[7]^{1)}$

$$
\mathbf{R}\left[\bar{\alpha}_{s}^{(1)}\right]=\tilde{\alpha}^{(1)}(s)=\frac{1}{\beta_{0}}\left[\frac{1}{2}-\frac{1}{\pi} \operatorname{arctg} \frac{L}{\pi}\right]_{L>0}=\frac{1}{\beta_{0} \pi} \operatorname{arctg} \frac{\pi}{L}, \quad L=\ln \frac{s}{\Lambda^{2}} .
$$

2. В двухпетлевом случае популярному приближению

$$
\beta_{0} \bar{\alpha}_{s, \mathrm{pop}}^{(2)}\left(Q^{2}\right)=\frac{1}{l}-b_{1}(f) \frac{\ln l}{l^{2}}, \quad l=\ln \frac{Q^{2}}{\Lambda^{2}},
$$

\footnotetext{
1) В дальнейшем эта идея неоднократно обсуждалась рядом известных авторов (см. [10]-[15]).
} 
отвечает [7], [15]

$$
\tilde{\alpha}_{\text {pop }}^{(2)}(s)=\left(1+\frac{b_{1} L}{L^{2}+\pi^{2}}\right) \tilde{\alpha}^{(1)}(s)-\frac{b_{1}}{\beta_{0}} \frac{\ln \left[\sqrt{L^{2}+\pi^{2}}\right]+1}{L^{2}+\pi^{2}} .
$$

Выражения (2) и (3) являются монотонно убываюшими с конечным инфракрасным (ИК) пределом $\tilde{\alpha}(0)=1 / \beta_{0}(f=3) \simeq 1.4$.

3. Квадрат и куб $\bar{\alpha}_{s}^{(1)}$ переходят в простые выражения [7], [8]

$$
\mathfrak{A}_{2}^{(1)}(s) \equiv \mathbf{R}\left[\left(\bar{\alpha}_{s}^{(1)}\right)^{2}\right]=\frac{1}{\beta_{0}^{2}\left[L^{2}+\pi^{2}\right]}, \quad \mathfrak{A}_{3}^{(1)}(s) \equiv \mathbf{R}\left[\left(\bar{\alpha}^{(1)}\right)^{3}\right]=\frac{L}{\beta_{0}^{3}\left[L^{2}+\pi^{2}\right]^{2}},
$$

не являющиеся степенями $\tilde{\alpha}^{(1)}(s)$.

Отметим здесь, что переход от сингулярных $\bar{\alpha}_{s}$ и их степеней к пипизированным выражениям (т.е. операция $\mathbf{R}$ ) может быть выполнен [11], [13] с помошью дифференциального оператора

$$
\mathcal{R}=\frac{\sin \pi \mathcal{P}}{\pi \mathcal{P}}, \quad \mathcal{P}=Q^{2} \frac{d}{d Q^{2}},
$$

с подстановкой $Q^{2} \rightarrow s$, например $\mathcal{R} \bar{\alpha}_{s}\left(Q^{2}\right)=\tilde{\alpha}(s)$.

Наиболее замечательным фактом для всех представленных $\tilde{\alpha}(s)$ и $\mathfrak{A}_{2,3}(s)$ (а также и в более общем случае) является исчезновение логарифмического полюса ${ }^{2)}$, который "закрьвается" $\pi^{2}$-вкладом.

Кроме того, привычное степенное "евклидово" разложение теории возмушений

$$
D_{\mathrm{pt}}\left(Q^{2}\right)=1+\sum_{k \geqslant 1} d_{k} \bar{\alpha}_{s}^{k}\left(Q^{2}\right)
$$

по степеням обычной, просуммированной по РГ, инвариантной функции связи $\bar{\alpha}_{s}\left(Q^{2}\right)$, обладающей нефизическими особенностями в ИК-области при $Q^{2} \leqslant \Lambda_{3}^{2}$, в результате преобразования $\mathbf{R}$ во времениподобной области заменяется на разложение по нестепенной последовательности функций

$$
R_{\pi}(s) \equiv \mathbf{R}\left[D_{\mathrm{pt}}\left(Q^{2}\right)\right]=1+\sum_{k \geqslant 1} d_{k} \mathfrak{A}_{k}(s), \quad \mathfrak{A}_{k}(s)=\mathbf{R}\left[\bar{\alpha}_{s}^{k}\left(Q^{2}\right)\right],
$$

обладающее улучшенным свойством убывания последующих членов [7].

При этом первая из этих функций $\tilde{\alpha}(s)=\mathfrak{A}_{1}(s)$ является монотонной и при $s=0$ принимает конечное значение $\tilde{\alpha}(0)=1 / \beta_{0}$ (см. ниже рис. 1 ), равное соответствующему предельному значению $\alpha_{\mathrm{an}}(0)$ аналитизированной функции связи в евклидовой области. В то же время "старшие” функции, подобные (4), нового разложения в ИК-пределе обращаются в нуль: $\mathfrak{A}_{k \geqslant 2}(0)=0$.

С другой стороны, в ультрафиолетовой (УФ) области при $\ln \left(s / \Lambda^{2}\right) \gg \pi$, т.е. при $W \gg \Lambda e^{\pi / 2} \simeq 3$ ГэВ, функции $\tilde{\alpha}$ и $\mathfrak{A}_{k}$ могут быть представлены рядами по степеням

\footnotetext{
2)Этот фракт остался неотмеченным в упомянутых пионерских работах 80-х годов.
} 
параметра $\pi^{2} / L^{2}, L=\ln \left(s / \Lambda^{2}\right)$. Такие выражения иногда переформулируют в разложения по степеням $\alpha_{s}$. Например, в однопетлевом приближении

$$
\tilde{\alpha}^{(1)}(s) \simeq \frac{1}{\beta_{0} L}-\frac{\pi^{2}}{3 L^{2}}+\frac{\pi^{4}}{5 L^{4}} \simeq \bar{\alpha}_{s}^{(1)}(s)-\frac{\pi^{2} \beta_{0}^{2}}{3}\left(\bar{\alpha}_{s}^{(1)}(s)\right)^{3}+\frac{\pi^{4} \beta_{0}^{4}}{5}\left(\bar{\alpha}_{s}^{(1)}(s)\right)^{5} .
$$

Не вдаваясь в детали, отметим, что качественно функции $\mathfrak{A}_{k}$ оказьваются поразительно близкими к функциям нестепенного разложения наблюдаемых $\mathcal{A}_{k}$ в евклидовой области [18], возникаюшим в так называемой аналитической теории возмушений (см. ниже п. 1.3 и рис. 2$)^{3)}$. В частности, вблизи нуля высшие функции $\mathfrak{A}_{k \geqslant 3}$ также осциллируют и образуют асимптотическую последовательность в смысле Эрдейи.

Отметим еше, что, как видно из формул (2) и (4), однопетлевые пипизированные функции удовлетворяют рекуррентному соотношению $(d / d L) \mathfrak{A}_{k}^{(1)}(s)=-k \beta_{0} \mathfrak{A}_{k+1}^{(1)}(s)$, которое является полным аналогом соответствуюшего соотношения для обычной функции связи, т.е. однопетлевого дифференциального уравнения. Как было отмечено в работе [18], подобное соотношение имеет место и для аналитизированных функций $\mathcal{A}_{k}^{(1)}$.

Недавно в работе [17] было найдено двухпетлевое обобщение

$$
\frac{1}{k} \frac{d}{d L} \mathfrak{A}_{k}^{(2)}(s)=-\beta_{0} \mathfrak{A}_{k+1}^{(2)}(s)-\beta_{1} \mathfrak{A}_{k+2}^{(2)}(s),
$$

представляюшее собой (при $k=1$ ) аналог двухпетлевого дифференциального уравнения для $\bar{\alpha}_{s}$. Аналогичное соотношение можно получить и для аналитизированных функций $\mathcal{A}_{k}$.

Для обратного перехода от области Минковского к области Евклида следует воспользоваться преобразованием $\mathbf{D}$, введенным в (1). Совершенно очевидно, что мы не вернемся к исходной $\bar{\alpha}_{s}$ и рядам по ее степеням (5). Для того чтобы прояснить ситуацию, полезно обратиться к основам инвариантного аналитического подхода.

1.3. Пространственноподобная область. АТВ. В самом деле, с конца 50-х годов известен прием [19] устранения нефизических особенностей в евклидовой области путем комбинирования результатов РГ-суммирования с требованием аналитичности по переменной $Q^{2}$, совместной с представлением Челлена-Лемана для $\bar{\alpha}_{s}\left(Q^{2}\right)$. Этот прием в середине 90-х годов был приложен [20]-[22] к КХД и назван инвариантнымм аналитическим подходом (ИАП). Его систематическое развитие и применение к пертурбативным разложениям для наблюдаемых привело к формулировке аналитической теории возмушений (ATB) [23]. Напомним здесь кратко основные черты и свойства АТВ (см. также недавний обзор [24]).

Комбинируя три элемента:

1) обычную квантово-полевую теорию возмушений для инвариантной функции связи и наблюдаемых;

2) перенормируемость и РГ-инвариантность;

\footnotetext{
${ }^{3)} \mathrm{Cм.}$ также первоначальную редакцию данной работы [16]. Более подробная численная информация о функциях $\tilde{\alpha}, \alpha_{\mathrm{an}}, \mathfrak{A}_{2,3}$ и $\mathcal{A}_{2,3}$ содержится в недавнем исследовании Маградзе [17].
} 
3) общие принципы локальной КТП, такие как причинность, унитарность, пуанкаре-инвариантность и спектральность, в форме спектральных представлений типа Челлена-Лемана и Йоста-Лемана-Дайсона,

удается сформулировать ИАП для пертурбативной КХД, в котором центральную роль играет спектральная плотность.

Будучи извлечена из обычных РГ-улучшенных выражений теории возмущений, эта спектральная плотность с помощью представления Челлена-Лемана определяет $Q^{2}$-аналитичные, РГ-инвариантные выражения для эффективной РГ-инвариантной функции связи и пертурбативных (т.е. представимых в виде разложений по степеням функции связи) наблюдаемых в евклидовой области.

В частности, ИАП приводит к модифицированному выражению $\alpha_{\text {an }}\left(Q^{2}\right)$ для инвариантной функции связи, свободному от призрачных сингулярностей и обладающему повышенной устойчивостью относительно высших петель и схемной зависимости [21]-[29].

ИАП изменяет структуру разложения теории возмущений для наблюдаемых: вместо привычного степенного ряда в результате интегрального преобразования возникает нестепенное асимптотическое разложение [18] в смысле Эрдейи по последовательности специфических функций $\mathcal{A}_{k}\left(Q^{2} ; f\right)$ (определенных интегральным преобразованием через соответствуюшие спектральные плотности), которые свободны от нефизических особенностей. Подобное нестепенное разложение с коэффициентами, полученными из надлежащих диаграмм Фейнмана, мы и называем аналитической теорией возмущений.

Функции $\mathcal{A}_{k}$ при малых значениях $Q^{2}$ с ростом индекса $k$ убывают быстрее, чем соответствуюшие степени $\alpha_{\text {an }}$ (и даже осциллируют в области $Q \leqslant \Lambda$ ), вследствие чего свойства сходимости нового разложения сушественно улучшаются.

Основная цель этой работы состоит в прояснении соотношения между описанной выше процедурой суммирования $\pi^{2}$-членов (процедурой “пипизации” РКП), предложенной в работах [7], [8] и развитой затем Соловцовым с соавторами [12], [14] (фактически приводящей к определению инвариантной функции связи и ее эффективных степеней во времениподобной области), и АТВ в пространственноподобной области.

В результате подробного анализа мы расширяем АТВ на времениподобную область, напоминаем замечательный эффект "искривленного зеркала" [27] в корреляциях между аналитизированными и пипизированными эффективными функциями связи КХД - евклидовой $\alpha_{\text {an }}$ и минковской $\tilde{\alpha}$, и обнаруживаем соответствуюший эффект для высших функций нестепенных асимптотических разложений $\mathcal{A}_{k}$ и $\mathfrak{A}_{k}$ (см. ниже рис. 2).

В разделе 3 мы рассматриваем переход через порог рождения тяжелого кварка и конструируем "глобальную" АТВ, применимую во всей физической области $M_{\tau} \lesssim \sqrt{s}$, $Q \lesssim M_{Z}$

Здесь уместно отметить, что предыдушие работы 80-х и 90-х годов [20]-[26] и [28]-[35] подразумевали некоторое фиксированное число $f$ активных кварков. Глобальный случай отвечает более реалистической ситуации. Поэтому наши результаты нацелены на 
использование в анализе корреляции между результатами экспериментов, проведенных в различных областях энергий и переданных импульсов.

Основные результаты этого исследования кратко суммированы в разделе 5.

\section{2. САМОСОГЛАСОВАННАЯ СХЕМА ДЛЯ НАБЛЮДАЕМЫХ}

2.1. Модификация АТВ. Как было отмечено выше, применение операции $\mathbf{D}$ к эффективной функции связи $\tilde{\alpha}$ не возврашает нас к $\bar{\alpha}_{s}$, так как представление (1) несовместно с нефизической сингулярностью последней. Вместо этого мы приходим к

$$
\mathbf{D}\{\tilde{\alpha}(s ; f)\}=\frac{Q^{2}}{\pi} \int_{0}^{\infty} \frac{d s}{\left(s+Q^{2}\right)^{2}} \tilde{\alpha}(s ; f) \equiv \alpha_{\text {an }}\left(Q^{2} ; f\right),
$$

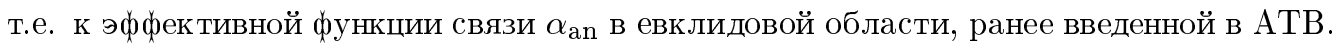
Этот простой факт впервые был установлен в работе [12]. Из него следует, что операции $\mathbf{D}$ и $\mathbf{R}$ связывают между собой пипизированные и аналитизированные функции связи в евклидовой и минковской областях. Таким образом, в данном случае $\mathbf{R}=\mathbf{D}^{-1}$. Видно, однако, что соотношение $\mathbf{D R}=\mathbf{1}$ имеет место лишь на классе функиий $F\left(Q^{2}\right)$, удовлетворяющих условию Челлена-Лемана.

Мы получаем возможность распространить АТВ на времениподобную область. Сделаем это в форме “рецептурного предписания", используя операцию аналитизации

$$
F\left(Q^{2}\right) \rightarrow F_{\text {an }}\left(Q^{2}\right)=\mathbf{A} \cdot F\left(Q^{2}\right),
$$

введенную в работах [20], [21] в терминах представления Челлена-Лемана

$$
F_{\mathrm{an}}\left(Q^{2}\right)=\frac{1}{\pi} \int_{0}^{\infty} \frac{d \sigma}{\sigma+Q^{2}} \rho_{\mathrm{pt}}(\sigma), \quad \rho_{\mathrm{pt}}(\sigma)=\Im F(-\sigma),
$$

причем по прескрипции спектральная плотность определяется на основе прямого аналитического продолжения рассматриваемой функции $F$ на разрез. Формулы $(10)$ и $(11)$ в совокупности определяют операцию аналитизации $\mathbf{A .}$

Теперь сформулируем АТВ. Во-первых, следует преобразовать обычную сингулярную функцию связи $\bar{\alpha}_{s}\left(Q^{2}\right)$ или разложение наблюдаемой по ее степеням

$$
D_{\mathrm{pt}}\left(Q^{2}\right)=1+\sum_{k \geqslant 1} d_{k} \bar{\alpha}_{s}^{k}\left(Q^{2} ; f\right)
$$

в соответствующее аналитизированное евклидово выражение $\alpha_{\text {an }}$ или $D_{\mathrm{an}}\left(Q^{2}\right)$, свободные от нефизических сингулярностей:

$$
\begin{aligned}
D_{\mathrm{an}}\left(Q^{2} ; f\right) & =1+\sum_{k \geqslant 1} d_{k} \mathcal{A}_{k}\left(Q^{2} ; f\right), & \alpha_{\mathrm{an}}\left(Q^{2} ; f\right) & =\mathcal{A}_{1}\left(Q^{2} ; f\right), \\
\mathcal{A}_{k}(x ; f) & =\frac{1}{\pi} \int_{0}^{\infty} \frac{d \sigma}{\sigma+x} \rho_{k}(\sigma ; f), & \rho_{k}(\sigma ; f) & =\Im\left[\bar{\alpha}_{s}^{k}(-\sigma ; f)\right],
\end{aligned}
$$

со спектральными плотностями $\rho, \rho_{k}$, введенными в соответствии с (11). 
Во-вторых, с помощью операции $\mathbf{R}$ мы определяем [14] во времениподобной области инвариантную функцию связи ${ }^{4)}$

$$
\alpha_{\text {an }}\left(Q^{2} ; f\right) \rightarrow \tilde{\alpha}(s ; f)=\mathbf{R}\left[\alpha_{\text {an }}\right]=\int_{s}^{\infty} \frac{d \sigma}{\sigma} \rho(\sigma ; f)
$$

или иную величину, например

$$
R_{\pi}(s) \equiv \mathbf{R}\left[D_{\mathrm{pt}}\left(Q^{2}\right)\right]=1+\sum_{k \geqslant 1} d_{k} \mathfrak{A}_{k}(s), \quad \mathfrak{A}_{k}(s)=\mathbf{R}\left[\bar{\alpha}_{s}^{k}\left(Q^{2}\right)\right],
$$

где

$$
\mathfrak{A}_{k}(s)=\int_{s}^{\infty} \frac{d \sigma}{\sigma} \rho_{k}(\sigma), \quad \rho_{k}(\sigma)=\Im\left[\alpha_{s}^{k}(-\sigma)\right] .
$$

Наконец, в-третьих, мы имеем возможность реконструкции евклидовых величин из соответствуюших минковских с помошью дипольного оператора $\mathbf{D}$. Так,

$$
\alpha_{\mathrm{an}}\left(Q^{2} ; f\right)=\mathbf{D}\{\tilde{\alpha}(s ; f)\} .
$$

В частности, подставляя $\tilde{\alpha}^{(1)}(s ; f)$ в подынтегральное выражение, получаем в результате интегрирования по частям

$$
\begin{aligned}
\mathbf{D}\left\{\tilde{\alpha}^{(1)}\right\} & =\frac{Q^{2}}{\pi \beta_{0}} \int_{0}^{\infty} \frac{d \sigma}{\left(\sigma+Q^{2}\right)^{2}}\left(\frac{1}{2}-\frac{1}{\pi} \operatorname{arctg} \frac{\ln \left(\frac{\sigma}{\Lambda^{2}}\right)}{\pi}\right)= \\
& =\frac{1}{\beta_{0}}\left[\frac{1}{\ln \frac{Q^{2}}{\Lambda^{2}}}+\frac{\Lambda^{2}}{\Lambda^{2}-Q^{2}}\right]=\alpha_{\text {an }}^{(1)}\left(Q^{2} ; f\right) .
\end{aligned}
$$

Это простое вычисление проясняет связь между свободными от призрачных сингулярностей выражениями в $s$ - и $Q^{2}$-каналах. Они связаны также и обратным преобразованием. Например, в соответствии с (15) имеем

$$
\tilde{\alpha}^{(1)}(s ; f)=\mathbf{R}\left[\alpha_{\text {an }}^{(1)}\left(Q^{2} ; f\right)\right] .
$$

На рис. 1 дана компактная сводка результатов АТВ для инвариантных аналитических функций связи $\alpha_{\text {an }}\left(Q^{2} ; 3\right)$ и $\tilde{\alpha}(s ; 3)$, вычисленных в одно-, двух- и трехпетлевых приближениях как в евклидовой, так и в минковской областях. Штрихпунктирные (верхние) кривые представляют однопетлевые приближения АТВ (2) и (17). Сплошными кривыми представлены двух- и трехпетлевые (в $\overline{\mathrm{MS}}$-схеме) приближения $\mathrm{ATB}^{5}$ ) , неразличимые между собой на нашем рисунке. Это замечательное совпадение (в пределах

\footnotetext{
4) Как следует из приведенного выражения, спектральная функция может играть роль бетафункции. Однако (в отличие от предположения, сделанного Швингером [36]) эта функция $\rho(s ; f)$, будучи спектральной функцией для евклидовой инвариантной связи, оказывается РГ-генератором для другой функции связи, определенной во времениподобной области [14].

${ }^{5)}$ Недавно было установлено [30], [31], что точное решение двухпетлевого РГ-уравнения для инвариантной функции связи $\bar{\alpha}_{s}\left(Q^{2}\right)$ может быть выражено через малоизвестную специальную функцию - функцию Ламберта $W$, определенную соотношением $W(z) e^{W(z)}=z$ и обладаюшую бесконечным числом ветвей $W_{n}(z)$. Через нее удается также представить [17], [30]-[33], [37] аналитизированные и пипизированные функции АТВ.
} 


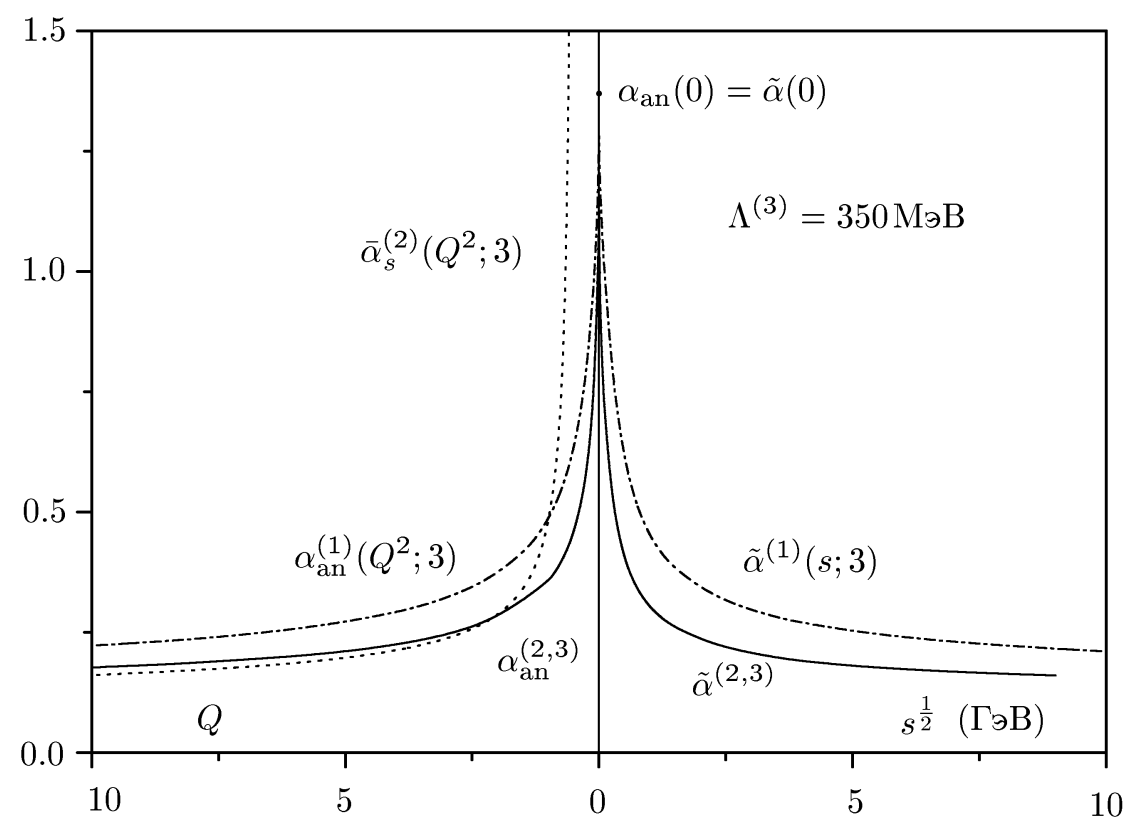

Рис.1. Пространственно- и времениподобные аналитические функции связи в области нескольких ГэВ.

1-2\%) демонстрирует характерную для АТВ пониженную чувствительность по отношению к высшим петлям (а также к изменению схемы перенормировки) как в евклидовой, так и в минковской областях от ИК- до УФ-пределов. Для сравнения в левой части рисунка пунктирной кривой изображена обычная функция $\bar{\alpha}_{s}\left(Q^{2}\right)$ в двухпетлевом приближении с особенностью при $Q^{2}=\Lambda^{2}$.

Как было установлено в работах [21], [25], [24], соотношения, аналогичные (15) и (9), имеют место для эффективных “степеней” инвариантной функции связи $\mathfrak{A}_{k}$ и $\mathcal{A}_{k}$. В целом мы получаем самосогласованную схему. Рассмотрим подробнее новые базисы разложения нестепенной теории возмущений.

2.2. Разложения по последовательностям $\{\mathcal{A}\}$ и $\{\mathfrak{A}\}$. Для более полного понимания следствий перехода от разложения по “традиционной" степенной последовательности

$$
\left\{\bar{\alpha}_{s}^{k}\left(Q^{2} ; f\right)\right\}=\bar{\alpha}_{s}\left(Q^{2}\right), \bar{\alpha}_{s}^{2}, \ldots, \bar{\alpha}_{s}^{k}, \ldots
$$

к разложениям по новым наборам функций в евклидовой и минковской областях

$$
\left\{\mathcal{A}_{k}\left(Q^{2} ; f\right)\right\}=\alpha_{\text {an }}\left(Q^{2} ; f\right), \mathcal{A}_{2}\left(Q^{2} ; f\right), \mathcal{A}_{3}, \ldots, \quad\left\{\mathfrak{A}_{k}(s ; f)\right\}=\tilde{\alpha}(s ; f), \mathfrak{A}_{2}(s ; f), \mathfrak{A}_{3}, \ldots
$$

полезно отметить некоторые свойства этих функций.

Оба нестепенных набора в определенном смысле подобны друг другу:

- Они состоят из убывающей последовательности функций, свободных от нефизических сингулярностей. 
- Первые члены обоих наборов, новые эффективные функции связи $\mathcal{A}_{1}=\alpha_{\text {an }}$ и $\mathfrak{A}_{1}=$ $\tilde{\alpha}$, оказываются монотонными функциями. В ИК-пределе они конечны и равны друг другу: $\alpha_{\text {an }}(0 ; 3)=\tilde{\alpha}(0 ; 3) \simeq 1.4$, и обладают бесконечными первыми производными. Обе имеют тот же самый лидируюший член $\sim 1 / \ln x$ в УФ-асимптотике.

- Все остальные функции ( "эффективные степени функций связи”) обоих наборов обрашаются в нуль в ИК-пределе: $\mathcal{A}_{k \geqslant 2}(0 ; f)=\mathfrak{A}_{k \geqslant 2}(0 ; f)=0$, и обладают УФ-поведением $\sim 1 /(\ln x)^{k}$, соответствуюшим $\bar{\alpha}_{s}^{k}(x)$. Они не являются монотонными. Вторые функции $\mathcal{A}_{2}$ и $\mathfrak{A}_{2}$ положительны с максимумом, лежашим вблизи $s, Q^{2} \sim \Lambda^{2}$. Высшие функции $\mathcal{A}_{k \geqslant 3}$ и $\mathfrak{A}_{k \geqslant 3}$ осциллируют в области малых значений аргументов и обладают $k-2$ нулями.

Замечательным образом механизмы “освобождения" от нефизических особенностей выглядят совершенно различными в евклидовой и минковской областях. В то время как в пространственноподобной области функции $\mathcal{A}_{k}$ являются аналитическими за счет аддитивных непертурбативных степенных по $Q^{2}$ структур, функции $\mathfrak{A}_{k}$ во времениподобной области оказываются несингулярными лишь за счет пересуммирования $\pi^{2}$-членов. Образно говоря, непертурбативная аналитизация в $Q^{2}$-канале является количественно искаженным отражением (операция $Q^{2} \rightarrow s=-Q^{2}$ ) внешне вполне пертурбативной “пипизации” в $s$-канале. Этот эффект “искривленного зеркала”, впервые обсуждавшийся в работе [27], проиллюстрирован на рис. 1 и 2 (см. ниже).

Подытожим полученные результаты, существенные для анализа данных. Вместо степенного ряда теории возмущений в евклидовой $D_{\mathrm{pt}}\left(Q^{2}\right)=1+d_{\mathrm{pt}}\left(Q^{2}\right)$,

$$
d_{\mathrm{pt}}\left(Q^{2}\right)=\sum_{k \geqslant 1} d_{k} \bar{\alpha}_{s}^{k}\left(Q^{2} ; f\right)
$$

и минковской областях $R_{\mathrm{pt}}(s)=1+r_{\mathrm{pt}}(s)$,

$$
r_{\mathrm{pt}}(s)=\sum_{k \geqslant 1} r_{k} \tilde{\alpha}^{k}(s ; f), \quad r_{1,2}=d_{1,2}, \quad r_{3}=d_{3}-d_{1} \frac{\pi^{2} \beta_{[f]}^{2}}{3}, \quad r_{4}=d_{4}-\cdots
$$

следует использовать асимптотические разложения (13) и (6)

$$
d_{\mathrm{an}}\left(Q^{2}\right)=\sum_{k \geqslant 1} d_{k} \mathcal{A}_{k}\left(Q^{2} ; f\right), \quad r_{\pi}(s)=\sum_{k \geqslant 1} d_{k} \mathfrak{A}_{k}(s ; f)
$$

с теми же самыми коэффициентами $d_{k}$ по нестепенным последовательностям функций $\{\mathcal{A}\}$ и $\{\mathfrak{A}\}$.

\section{3. ГЛОБАЛЬНАЯ ФОРМУЛИРОВКА АТВ}

Чтобы применить излагаемую схему к анализу процессов КХД, следует сформулировать ее "глобально" во всей области, доступной опыту, т.е. для областей с различными значениями числа $f$ активных кварков. Для этого следует обратиться к вопросу перехода через порог рождения нового кварка. 
3.1. Сопряжение на порогах. В реальных вычислениях используется процедура сопряжения на порогах. Одним из наиболее простых является условие сопряжения в безмассовой $\overline{\mathrm{MS}}$-схеме [38]

$$
\bar{\alpha}_{s}\left(Q^{2}=M_{f}^{2} ; f-1\right)=\bar{\alpha}_{s}\left(Q^{2}=M_{f}^{2} ; f\right),
$$

отнесенное к квадрату массы $M_{f}^{2} f$-го кварка.

$\mathrm{C}$ помощью условия непрерывности вводят "глобальную" функцию связи КХД $\bar{\alpha}_{s}\left(Q^{2}\right)$, составленную из гладких частей

$$
\bar{\alpha}_{s}\left(Q^{2}\right)=\bar{\alpha}_{s}\left(Q^{2} ; f\right) \quad \text { при } \quad M_{f-1}^{2} \leqslant Q^{2} \leqslant M_{f}^{2}
$$

и непрерывную во всем бесконечном интервале положительных евклидовых значений $Q^{2}$ с разрывами производных в точках сопряжения. Подобные функции можно называть сплайнами (первого порядка). На первый взгляд, любое безмассовое сопряжение, приводяшее к сплайну, уничтожая аналитичность по переменной $Q^{2}$, нарушает соответствие между $s$ - и $Q^{2}$-каналами ${ }^{6)}$.

Однако в рамках АТВ первоначальный степенной ряд теории возмущений (12) с его нефизическими сингулярностями и возможной неаналитичностью на порогах не имеет прямого отношения к физическим наблюдаемым, представляя собой лишь “сырье” для определения спектральной плотности. Между тем разрывность функции $\rho(\sigma)$ не представляет какой-либо опасности. В самом деле, выражение вида

$$
\rho_{k}(\sigma)=\rho_{k}(\sigma ; 3)+\sum_{f \geqslant 4} \theta\left(\sigma-M_{f}^{2}\right)\left\{\rho_{k}(\sigma ; f)-\rho_{k}(\sigma ; f-1)\right\},
$$

где $\rho_{k}(\sigma ; f)=\Im \bar{\alpha}_{s}^{k}(-\sigma ; f)$, определяет согласно (14) и (16) гладкую глобальную

$$
\mathcal{A}_{k}\left(Q^{2}\right)=\frac{1}{\pi} \int_{0}^{\infty} \frac{d \sigma}{\sigma+x} \rho_{k}(\sigma)
$$

и сплайн-непрерывную глобальную

$$
\mathfrak{A}_{k}(s)=\int_{s}^{\infty} \frac{d \sigma}{\sigma} \rho_{k}(\sigma)
$$

функции ${ }^{7)}$.

Видно, что в этой глобальной схеме исходная инвариантная функция связи $\bar{\alpha}_{s}\left(Q^{2}\right)$ играет двоякую роль. Она дает не просто спектральную плотность (20), но также и условие сопряжения (18), связывающее $\Lambda_{f}$ с $\Lambda_{f+1}$.

Заметим еще, что условие сопряжения на порогах (18) тесно связано с процедурой перенормировки [38], [40]. Именно по этой причине мы принимаем его в обычном виде (cp. c $[27])$.

\footnotetext{
6) Любая безмассовая схема является приближением, точность которого может быть проконтролирована соответствующей массовой схемой [39]. С помощью последней удается получить [40] плавный переход через порог рождения тяжелого кварка. Тем не менее с практической точки зрения достаточно использовать сплайновое условие сопряжения (18) (за исключением случаев, когда исследуется малая окрестность порога).

7) Формулами (20)-(22) мы ввели новые "глобальные" инвариантные эффективные функции связи и высшие функции, отличные от предыдущих с фиксированным значением $f$.
} 
3.2. $s$-Канал. Константы сдвига. С практической точки зрения сушественно, что “глобальная" $s$-канальная функция связи $\tilde{\alpha}(s)$, равно как и высшие функции $\mathfrak{A}_{k}(s)$, вообще говоря, отличаются на константы от их аналогов $\tilde{\alpha}(s ; f)$ и $\mathfrak{A}_{k}(s ; f)$ с фиксированным значением числа $f$. Например, при $M_{5}^{2} \leqslant s \leqslant M_{6}^{2}$

$$
\tilde{\alpha}(s)=\int_{s}^{\infty} \frac{d \sigma}{\sigma} \rho(\sigma)=\int_{s}^{M_{6}^{2}} \frac{d \sigma}{\sigma} \rho(\sigma ; 5)+\int_{M_{6}^{2}}^{\infty} \frac{d \sigma}{\sigma} \rho(\sigma ; 6)=\tilde{\alpha}(s ; 5)+c(5) .
$$

В обшем случае имеем

$$
\tilde{\alpha}(s)=\tilde{\alpha}(s ; f)+c(f) \quad \text { при } \quad M_{f}^{2} \leqslant s \leqslant M_{f+1}^{2}
$$

с константами сдвига $c(f)$, представимыми через интегралы, содержащие плотности $\rho(\sigma ; f+n), n \geqslant 1$, при дополнительном условии $c(6)=0$, отвечаюшем асимптотической свободе. Именно,

$$
c(f-1)=\tilde{\alpha}\left(M_{f}^{2} ; f\right)-\tilde{\alpha}\left(M_{f}^{2} ; f-1\right)+c(f), \quad c(6)=0 .
$$

Эти константы $c(f)$ обеспечивают непрерьвность $\tilde{\alpha}(s)$ в точках сопряжения $M_{f}^{2}$.

Аналогичные константы сдвига

$$
\mathfrak{A}_{k}(s)=\mathfrak{A}_{k}(s ; f)+\mathfrak{c}_{k}(f) \quad \text { при } \quad M_{f}^{2} \leqslant s \leqslant M_{f+1}^{2}
$$

ответственны за непрерывность высших функций $\mathfrak{A}_{k}$.

В то же время константы $\mathfrak{c}_{2}(f)$ описывают скачки "главной" спектральной функции $(20)$.

Однопетлевая оценка

$$
c(f-1)-c(f)=\frac{1}{\pi \beta_{[f]}} \operatorname{arctg} \frac{\pi}{\ln \frac{M_{f}^{2}}{\Lambda_{f}^{2}}}-\frac{1}{\pi \beta_{[f-1]}} \operatorname{arctg} \frac{\pi}{\ln \frac{M_{f}^{2}}{\Lambda_{f-1}^{2}}} \simeq \frac{17-f}{54} \bar{\alpha}_{s}^{3}\left(M_{f}^{2}\right)
$$

с использованием $\beta_{[f]} \rho(\sigma ; f)=\left\{\ln ^{2}\left(\sigma / \Lambda_{f}^{2}\right)+\pi^{2}\right\}^{-1}$ и с принятыми значениями параметров $\Lambda_{3}, \Lambda_{4} \sim 350-250$ МэВ показывает, что эти константы

$$
c(5) \simeq 3 \cdot 10^{-4}, \quad c(4) \simeq 3 \cdot 10^{-3}, \quad c(3) \simeq 0.01, \quad \mathfrak{c}_{2}(f) \simeq 3 \alpha\left(M_{f}^{2}\right) c(f)
$$

могут быть сушественны на уровне нескольких процентов для $\tilde{\alpha}$ и на уровне $\sim 10 \%$ для $\mathfrak{A}_{2}$.

В свою очередь, это означает, что количественный анализ $s$-канальных экспериментов в области $f=3$, например $e^{+} e^{-}$-аннигиляции в адроны [24], $\tau$-лептонного распада [25] и ширины чармония [8], может быть модифицирован с учетом этих констант. 
3.3. Глобальные евклидовы функции. В то же время в евклидовой области вместо первоначальной сплайновой функции $\bar{\alpha}_{s}$ мы имеем теперь гладкую, аналитическую во всей области $Q^{2}>0$, глобальную инвариантную функцию связи, определенную подобно (21) через спектральный интеграл

$$
\alpha_{\text {an }}\left(Q^{2}\right)=\frac{1}{\pi} \int_{0}^{\infty} \frac{d \sigma}{\sigma+Q^{2}} \rho(\sigma)
$$

с разрывной плотностью $\rho(\sigma)(20)$.

$\mathrm{K}$ сожалению, здесь, в отличие от времениподобного случая, не представляется возможным получить явное выражение для $\alpha_{\mathrm{an}}\left(Q^{2}\right)$ даже в однопетлевом случае. Более того, все евклидовы функции $\alpha_{\text {an }}$ и $\mathcal{A}_{k}$, рассматриваемые в интервале $M_{f}^{2} \leqslant Q^{2} \leqslant M_{f+1}^{2}$, отвечающем некоторому частному значению числа $f$, зависят от значений всех четырех параметров $\Lambda_{3}, \ldots, \Lambda_{6}$ одновременно. Тем не менее в действительности численная разница со случаем $f=3$ невелика при малых $Q^{2}$ и в области нескольких ГэВ несушественна.

Положение иллюстрируется рис. 2. Здесь толстыми сплошными кривыми с максимумом в окрестности $\sqrt{s}, Q \equiv \Lambda$ изображены вторые функции $\mathcal{A}_{2}$ и $\mathfrak{A}_{2}$ в области нескольких ГэВ. Тонкими сплошными кривыми с нулями в окрестности $\Lambda$ и отришательными значениями представлены функции $\mathcal{A}_{3}$ и $\mathfrak{A}_{3}$. Для сравнения приведены также вторые и третьи степени соответствуюших эффективных функций $\alpha_{\text {an }}$ и $\tilde{\alpha}$.

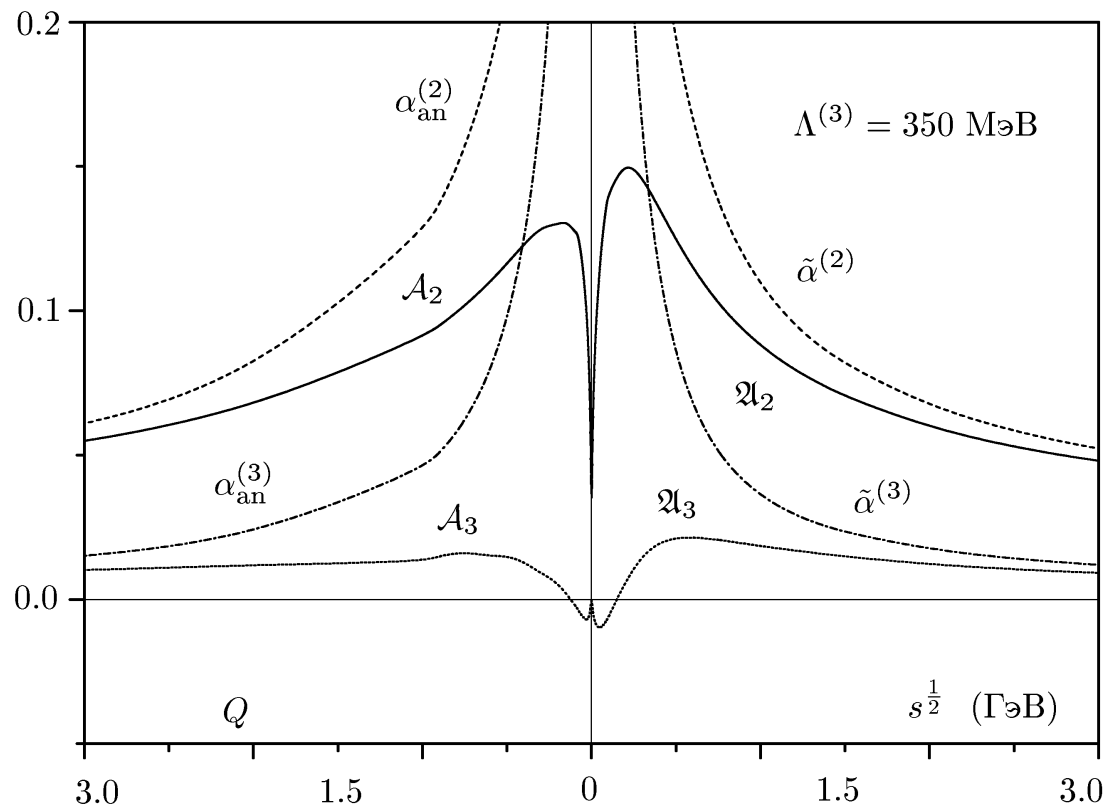

Рис. 2. "Искаженная зеркальная симметрия" для высших функций разложения. 
Все эти кривые соответствуют двухпетлевому случаю и вычислены на основе точных решений, представленных через функции Ламберта ${ }^{8)}$.

\section{4. ИЛЛЮСТРАЦИИ}

Важный количественный эффект при извлечении значений эффективной функции связи возникает из-за нестепенного характера разложения теории возмущений в АТВ. Он особенно заметен в области нескольких ГэВ.

4.1. $s$-Канал. Для иллюстрации количественной разницы между глобальной схемой и обычной практикой обработки данных рассмотрим пару примеров.

Процесс инклюзивной $e^{+} e^{-}$-аннигиляции в адроны является важным источником получения параметров КХД. Обычно (см., например, [4]) основное соотношение представляют в форме

$$
\frac{R(s)}{R_{0}}=1+r(s), \quad r(s)=\frac{\bar{\alpha}_{s}(s)}{\pi}+r_{2} \bar{\alpha}_{s}^{2}(s)+r_{3} \bar{\alpha}_{s}^{3}(s) .
$$

Здесь численные коэффициенты $r_{1}=1 / \pi=0.318, r_{2}=0.142, r_{3}=-0.413$ (приведенные для $f=5$ ) оказываются неубываюшими. Однако большое отрицательное численное значение $r_{3}$ обусловлено в основном вкладом $-r_{1} \pi^{2} \beta_{[5]}^{2} / 3$, равным -0.456 .

В АТВ вместо (27) с учетом (6) мы имеем

$$
r(s)=1+\frac{\tilde{\alpha}(s)}{\pi}+d_{2} \mathfrak{A}_{2}(s)+d_{3} \mathfrak{A}_{3}(s)
$$

с разумно убываюшими коэффициентами: $d_{1}=0.318, d_{2}=0.142, d_{3}=0.043$. Упомянутый $\pi^{2}$-вклад из $r_{3}$ теперь “поглошен” в $\tilde{\alpha}(s)^{9)}$.

Теперь основное различие между соотношениями $(27)$ и (28) обусловлено разницей между вкладом $d_{2} \bar{\alpha}_{s}^{2}$ и заменившим его $d_{2} \mathfrak{A}_{2}$, которая в первом приближении может быть представлена структурой $r_{4} \alpha^{4}$, причем $r_{4}=d_{2} \beta_{[5]}^{2} \pi^{2} \simeq 0.62$. Этот эффект может быть заметным при $\tilde{\alpha}(s) \simeq \bar{\alpha}_{s}(s) \simeq 0.20-0.25$. Проиллюстрируем его на примеpe $e^{+} e^{-}$-аннигилящии при энергии $W=\sqrt{s_{1}}=10.5$ ГэВ. Согласно сводной таблице из обзора Бетке [5] $\bar{\alpha}_{s}\left(s_{1}\right)=0.200$, что эквивалентно $r\left(s_{1}\right)=0.066$. При обработке этого значения с помошью формулы (28) получим $\tilde{\alpha}\left(s_{1}\right)=0.192$, что примерно эквивалентно $\bar{\alpha}_{s}\left(s_{1}\right)=0.194$.

Примечательно, что третий (трехпетлевой) член в АТВ-анализе дает вклад на уровне половины процента против $5.5 \%$ при обычном анализе.

8) Детали вычислений представлены, например, в работе [17]. Они были проведены при участии Б. А. Маградзе и Д. С. Курашева. Свойства функций Ламберта можно найти в работе [37].

9) Этот член вносит вклад примерно $8 \cdot 10^{-4}$ в $r\left(M_{Z}^{2}\right)$ и соответственно 0.0025 в извлеченное значение $\bar{\alpha}_{s}\left(M_{Z}^{2}\right)$. Это, в частности, означает, что главная часть "традиционного трехпетлевого члена" $r_{3} \bar{\alpha}_{s}^{3}$ в правой части $(27)$, будучи однопетлевой природы, оказывается существенной для количественного анализа данных. В частности, ее следует учитывать при так называемом NLLA-приближении, которое часто используется при анализе данных в пятикварковой области при $\sqrt{s} \sim M_{Z}$. 
Инклюзивный $\tau$-распад. Алгоритм АТВ при фиксированном $f=3$ был недавно использован в работе [41] для анализа инклюзивного $\tau$-распада. Здесь наблюдаемая величина - время жизни $\tau$-лептона - содержит поправку $\Delta$ от сильных взаимодействий, выраженную через интеграл от $s$-канального матричного элемента по области $0<s<$ $M_{\tau}^{2}$. В результате трехпетлевого АТВ-анализа современного экспериментального значения $\Delta_{\exp }\left(s_{0}=3.16\right)=0.191[42]$ было получено $\tilde{\alpha}\left(M_{\tau}^{2}\right)=0.380$. Эту величину можно сравнить со значением $\bar{\alpha}_{s}\left(M_{\tau}^{2}\right)=0.334$, которое соответствует обработке того же значения при помощи обычного степенного трехчлена. Отметим еще, что при АТВ-обработке $\tau$-распада второй и третий члены теории возмушений существенно подавлены, так что третий член дает вклад $1 \%$.

4.2. $Q^{2}$-канал. Правила сумм. В работе [26] АТВ была применена к правилам сумм Бвёркена. Здесь приходится иметь дело с евклидовым случаем и малыми передачами импульса $Q^{2} \lesssim 10$ Гэ $\mathrm{B}^{2}$.

Ввиду некоторой противоречивости экспериментальных данных мы приведем лиш часть результатов упомянутого исследования. Например, используя данные коллаборации $\mathrm{SMC} \mathrm{[43]} \mathrm{при} Q_{0}^{2}=10$ Гэ $\mathrm{B}^{2}$, авторы [26] получили $\alpha_{\mathrm{an}}^{(3)}\left(Q_{0}^{2}\right)=0.301$ вместо значения $\alpha_{\mathrm{S}}^{(3)}\left(Q_{0}^{2}\right)=0.275$, типичного для стандартного анализа.

Напомним, что в АТВ для наблюдаемой вместо обычного степенного разложения вида (12) мы имеем дело с нестепенным разложением типа (13). Здесь модификация обусловлена непертурбативными степенными вкладами $\Lambda^{2} / Q^{2}$ при $Q^{2} \gg \Lambda^{2}$. Как было оценено выше, эти поправки могут быть сушественны в области значений передач импульса порядка нескольких ГэВ.

Это замечание может быть также отнесено к анализу правила сумм Гросса-Льюэллина-Смита (ГЛС) [28].

Отметим еше, что общей чертой использования АТВ в упомянутых правилах сумм является сушественное понижение роли вкладов высших петель. Так, для правил сумм ГЛС, вместо пропорции $(65: 24: 11){ }_{\text {ТВ }}{ }^{10)}$ обычного анализа, АТВ

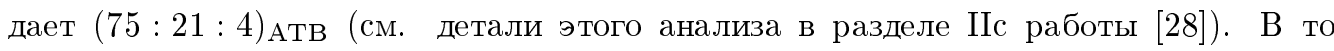
же время для правил сумм Бьёркена эффект оказывается еше более значительным $(55: 26: 19)_{\mathrm{TB}} \rightarrow(80: 19: 1)_{\text {АТв }}[26]$.

Сделаем еще пару комментариев.

1. Как видно, значения $\alpha_{\text {an и }} \tilde{\alpha}$, извлекаемые методом АТВ из тех же самых данных, оказываются несколько большими соответствуюших значений $\bar{\alpha}_{s}$, получаемых обычным путем. Причина лежит в нестепенном характере новых асимптотических разложений, в которых вклады высших петель оказываются заметно подавленными в области нескольких ГэВ.

2. В то же время для равных значений $\alpha_{\text {аn }}\left(x_{*}\right)=\tilde{\alpha}\left(x_{*}\right)=\bar{\alpha}_{s}\left(x_{*}\right)$ значения параметра шкалы $\mathrm{KX}$ Д $\Lambda_{\mathrm{an}}$, извлеченного из $\alpha_{\text {an }}$ и $\tilde{\alpha}$, могут оказаться больше значения $\Lambda_{\overline{\mathrm{MS}}}$, полученного из $\bar{\alpha}_{s}$. Последнее свойство обусловлено более “гладким" поведением функций $\alpha_{\text {an }}$ и $\tilde{\alpha}$ по сравнению с регулярной $\bar{\alpha}_{s}$.

${ }^{10)}$ То есть вклад первого, линейного по $\bar{\alpha}_{s}$, члена составляет $65 \%$, а вклады двух- и трехпетлевого - 24 и $11 \%$, соответственно. 


\section{5. ЗАКЛЮЧЕНИЕ}

Сформулируем еше раз основные утверждения АТВ и полученные результаты.

1. В рамках пертурбативной КХД построена самосогласованная схема для анализа данных как в пространственно-, так и во времениподобной областях, свободная от нефизических сингулярностей. Основными соотношениями, связывающими эти области, являются дипольное спектральное преобразование (1) и обратное ему, трансформирующие инвариантные нестепенные разложения $D_{\text {an }}\left(Q^{2}\right)$ и $R_{\pi}(s)$ друг в друга. Именно уравнение (1), эквивалентное представлению Челлена-Лемана, рассматриваемое как преобразование, ответственно за непертурбативные слагаемые в евклидовых выражениях для $\alpha_{\text {an }}\left(Q^{2}\right)$ и функции разложения $\left\{\mathcal{A}_{k}\left(Q^{2}\right)\right\}$. Таким образом, эти неаналитические по константе связи $\alpha$ вклады соответствуют вполне пертурбативным $\pi^{2}$-членам, эффективно просуммированным в $s$-канальные выражения $\tilde{\alpha}(s)$ и $\mathfrak{A}_{k}(s)$.

2. В качестве побочного результата мы установили новое качественное свойство АТВ, связанное с непертурбативностью в евклидовой области, которую следует рассматривать как минимальную непертурбативность или минимальную неаналитичность ${ }^{11)}$ по $\alpha$, поскольку ей отвечает пертурбативность в $s$-канале.

Следует добавить, что минимальная непертурбативность не может отвечать какому-либо физическому механизму; в противном случае придется допустить, что такой механизм "не оставляет следов" в $s$-канале.

3. Следуюший результат относится к корреляции между областями с различными значениями эффективного числа ароматов $f$. Имея в виду безмассовую $\overline{\mathrm{MS}}$-схему перенормировки, мы обрашаем внимание на то, что обычное разложение теории возмушений КХД дает нам разрьвную спектральную плотность (20), одновременно зависяшую от нескольких параметров шкалы $\Lambda_{f}, f=3, \ldots, 6$, связанных между собой обычными условиями сопряжения (18). Эта разрывная плотность приводит, с одной стороны, к гладкой евклидовой эффективной функции связи $\alpha_{\mathrm{an}}\left(Q^{2}\right)$ и высшим функциям $\left\{\mathcal{A}_{k}\left(Q^{2}\right)\right\}$ в пространственноподобной области (см. (21)). С другой стороны, она дает сплайн-непрерывные эффективную инвариантную связь $\tilde{\alpha}(s)$ и высшие функции $\left\{\mathfrak{A}_{k}(s)\right\}$ во времениподобной области (см. (22)). Глобальные функции $\left\{\mathcal{A}_{k}\left(Q^{2}\right)\right\}$ и $\left\{\mathfrak{A}_{k}(s)\right\}$ отличаются от их аналогов $\left\{\mathcal{A}_{k}\left(Q^{2} ; f\right)\right\}$ и $\left\{\mathfrak{A}_{k}(s ; f)\right\}$ с фиксированным значением числа ароматов.

4. Таким образом, новая глобальная схема АТВ использует обычную инвариантную функцию связи $\bar{\alpha}_{s}\left(Q^{2} ; f\right)$ и обычные условия сопряжения на порогах только в качестве исходного теоретического материала. В то же время практические вычисления для наблюдаемых основаны на разложениях по последовательностям $\left\{\mathcal{A}_{k}\left(Q^{2}\right)\right\}$ и $\left\{\mathfrak{A}_{k}(s)\right\}$, т.е. на нестепенных рядах с обычными численными коэффициентами $d_{k}$, полученными из анализа соответствуюших диаграмм Фейнмана. Это означает, в частности, что в на-

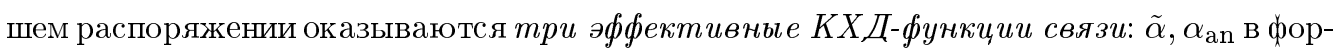
мализме $\mathrm{ATB}$, а также традиционная функция $\bar{\alpha}_{s}$, через которую можно приближенно выражать результаты, полученные в терминах двух предыдущих, в четырех- и пятикварковых областях в целях сравнения с данными обычного анализа.

\footnotetext{
${ }^{11)}$ Совместную с РГ-инвариатностью и $Q^{2}$-аналитичностью (ср. с [44]).
} 
В то же время следует подвергнуть анализу в рамках АТВ основную массу результатов по извлечению параметров КХД из низкоэнергетических экспериментов. Наши предварительные оценки показали, что подобная ревизия может повлиять на степень их соответствия друг другу.

5. Последнее по порядку, но не по важности. Как было отмечено в наших недавних обзорах [21], [24], АТВ обладает "повышенным иммунитетом" по отношению к учету высших петель и изменению схемы перенормировки.

На основе полученного в этой работе материала по разложениям для наблюдаемых можно констатировать, что разложения АТВ имеют лучшие свойства сходимости по сравнению с обычной РГ-просуммированной теорией возмущений как в $s$-, так и в $Q^{2}$-каналах

Благодарности. Автор признателен Д. Ю. Бардину, А. В. Ефремову, Н. В. Красникову, Д. С. Курашеву, Б. А. Маградзе, С. В. Михайлову, А. В. Радюшкину, И. Л. Соловцову, О.П. Соловцовой и Никосу Стефанису за полезные обсуждения и замечания. Это исследование частично поддержано грантами РФФИ № 99-01-00091 и 00-15-96691, грантом INTAS № 96-0842 и грантом INTAS-CERN № 2000-377, а также грантом программы Гайзенберг-Ландау.

\section{Список литературы}

[1] Н. Н. Боголюбов, Д. В. Ширков. ДАН СССР. 1955. Т. 103. С. 203; ЖЭТФ. 1956. Т. 30. C. 77 ; N. N. Bogolubov, D. V. Shirkov. Nuovo Cimento. 1956. V. 3. P. 845.

[2] P.A.M. Dirac. In: Theorie du Positron (7-eme Conseil du Physique Solvay: Structure et propriete de noyaux atomiques, Oct.1933). Paris: Gauthier-Villars, 1934. P. 203.

[3] Н. Н. Боголюбов, Д. В. Ширков. Введение в теорию квантованных полей. М.: ГТИ, 1957; М.: Наука 1973, 1976, 1984.

[4] D. E. Groom et al. Eur. Phys. J. C. 2000. V. 15. P. 1.

[5] S. Bethke. J. Phys. G. 2000. V. 26. P. R27; hep-ex/0004021.

[6] D. Yu. Bardin, G. Passarino. The Standard model in the Making. Oxford: Clarendon Press, 1999.

[7] A.V. Radyushkin. Optimizated $\Lambda$-parametrization for the QCD running coupling constant in space like and time like regions. JINR preprint E2-82-159; А. В. Радюшкин. Кратк. сообщ. ОИЯИ. 1996. № 4[78]-96. С. 9; hep-ph/9907228.

[8] N. V. Krasnikov, A. A. Pivovarov. Phys. Lett. B. 1982. V. 116. P. 168.

[9] B. Schrempp, F. Schrempp. Z. Phys. C. 1980. V. 6. P. 7.

[10] J. D. Bjorken. Two topics in QCD. Preprint SLAC-PUB-5103 (Dec. 1989); In: Proc. Cargese Summer Institute. Nato Adv. Inst. Ser. B. V.223. Eds. M. Levy et al. N.Y.: Plenum Press, 1990.

[11] A. L. Kataev, V. V. Starshenko. Mod. Phys. Lett. A. 1995. V. 19. P. 235.

[12] H. F. Jones, I. L. Solovtsov. Phys. Lett. B. 1995. V. 349. P. 519.

[13] Yu. L. Dokshitzer et al. Nucl. Phys. B. 1996. V. 469. P. 93.

[14] K. A. Milton, I. L. Solovtsov. Phys. Rev. D. 1997. V. 55. P. 5295.

[15] A.P. Bakulev, A.V. Radyushkin, N. G. Stefanis. Form Factors and QCD in spacelike and timelike regions. hep-ph/0005085.

[16] D. V. Shirkov. Toward the correlated analysis of perturbative QCD observables. JINR preprint E2-2000-46; hep-ph/0003242.

[17] B. V. Magradze. QCD coupling up to 3rd order in standard and analytic perturbation theories. JINR preprint E2-2000-222; hep-ph/0010070. 
[18] D. V. Shirkov. Lett. Math. Phys. 1999. V. 48. Р. 135; Д. В. Ширков. ТМФ. 1999. Т. 119. C. $55 ;$ hep-th $/ 9810246$.

[19] Н. Н. Боголюбов, А. А. Логунов, Д. В. Ширков. ЖЭТФ. 1959. Т. 37. С. 805.

[20] Д. В. Ширков, И. Л. Соловцов. Кратк. сообш. ОИЯИ. 1996. № 2[76]-96. С. 5; hep-ph/9604363.

[21] D. V. Shirkov, I. L. Solovtsov. Phys. Rev. Lett. 1997. V. 79. P. 1209; hep-ph/9704333.

[22] D. V. Shirkov, I. L. Solovtsov. Phys. Lett. B. 1998. V. 442. P. 344; hep-ph/9711251.

[23] D. V. Shirkov. Nucl. Phys. B. (Proceed. Suppl. ). 1998. V. 64. P. 106.

[24] И. Л. Соловцов, Д. В. Ширков. ТМФ. 1999. Т. 120. С. 482; hep-ph/9909305.

[25] K. A. Milton, I. L. Solovtsov, O. P. Solovtsova. Phys. Lett. B. 1997. V. 415. P. 104.

[26] K. A. Milton, I. L. Solovtsov, O.P. Solovtsova. Phys. Lett. B. 1998. V. 439. P. 421; hep-ph/9809510.

[27] K. A. Milton, O. P. Solovtsova. Phys. Rev. D. 1998. V. 57. P. 5402.

[28] K. A. Milton, I. L. Solovtsov, O.P. Solovtsova. Phys. Rev. D. 1999. V. 60. P. 016001; hep-ph/9809513.

[29] N. G. Stefanis, W. Schroers, H.-Ch. Kim. Phys. Lett. B. 1999. V. 449. P. 299; hep-ph/9812280.

[30] E. Gardi, G. Grunberg, M. Karliner. JHEP. 1998. V. 07. P. 007; hep-ph/9806462.

[31] B. A. Magradze. The gluon propagator in analytic perturbation theory. In: Proc. X Intern. Seminar "QUARKS-98" (Suzdal, Russia, May, 1998). V. 1. Eds. F.L. Besrukov et al. M.: INR Publ., 2000. P. 158; hep-ph/9808247.

[32] B. A. Magradze. An analytic approach to perturbative QCD. hep-ph/9911456; Int. J. Mod. Phys. A. 2000. V. 15. P. 2713.

[33] D. S. Kurashev. Exact analytic two-loop expressions for QCD observables in the time-like region. hep-th/0010072.

[34] A. A. Pivovarov. Z. Phys. C. 1992. V. 53. P. 461

[35] Б. В. Гешкенбейн, Б. Л. Иоффе. Письма в ЖЭТФ. 1999. Т. 70. С. 167.

[36] J. Schwinger. Proc. Nat. Acad. Sci. USA. 1974. V. 71. P. 3024; P. 5047.

[37] R. M. Corless et al. Adv. Comput. Math. 1996. V. 5. P. 329.

[38] W. Bernreuther, W. Wetzel. Nucl. Phys. B. 1982. V. 197. P. 228; W. Marciano. Phys. Rev. D. 1984. V. 29. P. 580.

[39] Д. В. Ширков. ТМФ. 1981. Т. 49. С. 291; D. V. Shirkov. Nucl. Phys. B. 1992. V. 371. P. 467.

[40] D. V. Shirkov, S. V. Mikhailov. Z. Phys. C. 1994. V. 63. P. 463.

[41] K. A. Milton, I. L. Solovtsov, O. P. Solovtsova, V. I. Yasnov. Eur. J. Phys. C. 2000. V. 14. P. 495; hep-ph/0003030.

[42] A. J. Weinstein. Nucl. Phys. B (Proc. Suppl. ). 1999. V. 76. P. 497.

[43] A. Adams et al. (SMC Collaboration). Phys. Rev. D. 1997. V. 56. P. 5330.

[44] D. V. Shirkov. Lett. Math. Phys. 1976. V. 1. P. 179; Lett. Nuovo Cimento. 1977. V. 18. P. 452. 